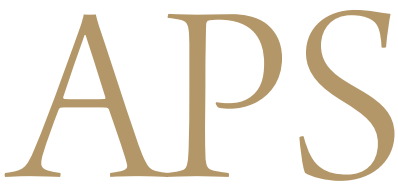

Archives of Plastic Surgery

\title{
Donor-Site Morbidity after Partial Second Toe Pulp Free Flap for Fingertip Reconstruction
}

\author{
Hyung Su Kim ${ }^{1}$, Dong Chul Lee ${ }^{1}$, Jin Soo Kim ${ }^{1}$, Si Young Roh ${ }^{1}$, Kyung Jin Lee ${ }^{1}$, Jae Won Yang ${ }^{2}$, \\ Sae Hwi Ki ${ }^{3}$, Aram Harijan ${ }^{4}$ \\ ${ }^{1}$ Department of Plastic and Reconstructive Surgery, Gwangmyeong Sungae General Hospital, Gwangmyeong; ${ }^{2}$ Plastic Surgery, Gangnam \\ Jaejun Plastic Clinic for Hand and Micro, Pyeongtaek; ${ }^{3}$ Department of Plastic and Reconstructive Surgery, Inha University School of Medicine, \\ Incheon; ${ }^{4}$ Well Plastic Surgery, Seoul, Korea
}

Background In this study, we characterize the morbidity at the donor-site of partial second toe pulp free flaps in terms of wound management as well as long-term outcomes.

Methods A single-institutional retrospective review was performed for patients who had undergone partial second toe pulp free flap transfer to the fingertip. Patient charts were reviewed for infection, skin necrosis, wound dehiscence, and hematoma for the donor site. Additionally, a questionnaire survey was given to patients who had a follow-up of longer than 1 year to characterize long-term postoperative pain and appearance.

Results The review identified a total of 246 cases. Early wound complications were significant for wound dehiscence $(n=8)$ and hematoma $(n=5)$ for a wound complication rate of $5.3 \%$. The questionnaire was distributed to 109 patients, and 54 patients completed the survey. Out of these 54 patients, 15 patients continued to have donor-site pain (28\%) at a mean followup period of 32.4 months. However, the pain intensity was relatively low in the range between 2 to 5 , on a 0-10 scale. None of these patients felt this donor-site pain interfered significantly with daily activity, nor did any patient require pain medications of any type. Donor-site appearance was satisfactory to most patients.

Conclusions The partial second toe pulp flap was associated with low rates of wound complications and favorable long-term outcomes. Given the functional and aesthetic gain in the recipient finger, donor-site morbidities appear acceptable in this patient population. This study can be helpful in counseling patients regarding donor-site morbidity during the informed consent process.

Keywords Free tissue flaps / Transplant donor site / Morbidity

\author{
Correspondence: Dong Chul Lee \\ Department of Plastic and \\ Reconstructive Surgery, \\ Gwangmyeong Sungae General \\ Hospital, 36 Digital-ro, Gwangmyeong \\ 14241, Korea \\ Tel: $+82-2-2680-7238$ \\ Fax: +82-2-2615-7218 \\ E-mail: ophand@gmail.com
}

No potential conflict of interest relevant to this article was reported.

Received: 15 Jun 2015 • Revised: 29 Jul 2015 • Accepted: 17 Aug 2015

pISSN: 2234-6163 • elSSN: 2234-6171 • http://dx.doi.org/10.5999/aps.2016.43.1.66• Arch Plast Surg 2016;43:66-70

\section{INTRODUCTION}

Fingertip defects are reconstructed using a variety of techniques [1-8]. For the past 15 years, our first-line treatment of such defects has been the partial second toe pulp free flap because it offers the replacement tissue with highest similarity to the lost pulp tissue with excellent sensory recovery, especially in young- er patients [1]. All reconstruction using autologous tissue has to take into account the trade off between donor-site morbidity and recipient-site gain. The primary goals of fingertip reconstruction using the toe pulp flap have been characterized fairly well, and we now turn surgeons' attention to donor-site morbidity. In this follow-up study, we attempt to describe immediate postoperative wound complications as well as long-term func- 
tional outcomes at the donor site.

\section{METHODS}

A single-institutional retrospective study was performed for all patients who had undergone partial second toe pulp free flap from January 2009 to August 2014 (Table 1). Patient charts and photographs were reviewed for demographic information, medical history (including smoking history), percentage of the second toe taken as a donor flap, and donor-site postoperative outcomes including complications. Early outcomes were evaluated for recorded instances of infection, skin necrosis, wound dehiscence, and hematoma for the donor site. Long-term outcomes were evaluated for sensory and nail deformities at follow-up lengths greater than 1 year. The Semmes-Weinstein monofilament test was used for the remaining toe pulp of the donor site and compared to the contralateral side as the control. A questionnaire survey was distributed to patients to evaluate general pain (11- point scale, $0-10)$, neuropathic pain (7-item DN4 score), need for pain medication, gait disturbance, and limitations in daily activity due to donor-site issues [9-11]. Moreover, the questionnaire included a patient-centric item assessing the patient's opinion on the appearance of the donor site on a 3-point scale (Table 2). Variables were assessed for statistical significance, using Fisher's exact test, the chi-squared test, or the independent t-test.

\section{Surgical technique}

The operative details regarding partial second toe pulp free flap have been described in a previous publication [1]. In brief, the pulp flap was designed in a teardrop shape on the medial side of

\section{Table 1. Measurements and records}

\begin{tabular}{|c|c|}
\hline Measurements & Records \\
\hline \multicolumn{2}{|l|}{ Early complications } \\
\hline Infection & Present/absent \\
\hline Skin necrosis & Present/absent \\
\hline Wound dehiscence & Present/absent \\
\hline Hematoma & Present/absent \\
\hline \multicolumn{2}{|l|}{ Long-term assessment } \\
\hline Sensory disturbance & $\begin{array}{l}\text { Normal/diminished light touch/diminished } \\
\text { protective sensation/loss of protective sensation }\end{array}$ \\
\hline Nail deformity & Present/Absent \\
\hline \multirow[t]{3}{*}{ Pain } & Present/Absent \\
\hline & Intensity self-evaluated on 11-point NRS \\
\hline & $\begin{array}{l}\text { Nature/associated situation/degree of effect on } \\
\text { daily activity }\end{array}$ \\
\hline Gait & Normal/abnormal \\
\hline \multicolumn{2}{|c|}{ Abnormality during daily activity Present/absent } \\
\hline Abnormality while working & Present/absent \\
\hline Appearance & Good/acceptable/poor \\
\hline
\end{tabular}

NRS, numerical rating scale (from 0, no pain; to 10, worst pain imaginable), the second toe (Fig. 1). Generally, the flaps were designed to contain less than $60 \%$ of the entire toe pulp arc (Fig. 2). After preserving the subcutaneous vein(s), the flap was dissected down to the pretendinous plane and elevated distally by dividing the vertical fibers. The neurovascular bundle supplying the medial pulp is isolated with a pedicle length of $2 \mathrm{~cm}$. Most of the donor sites are closed primarily, and any dog-ear was trimmed accordingly at the time of closure.

\section{RESULTS}

The retrospective search identified 246 cases of second toe pulp free flap over the 5-year period under review (Table 3). Donor sites had been closed primarily in all except one case, which was an exceptionally large toe pulp flap requiring a split-thickness skin graft for closure. Out of the total, wound complications had occurred in 13 cases (5.3\%). Eight of these were due to dehiscence after suture removal and were allowed to heal by secondary intention. The remaining five cases represented donor-site hematomas requiring evacuation and repeated closure. No wound infection or skin necrosis was observed. Neither pre-existing medical condition (hypertension or diabetes) nor smoking history correlated significantly with existence of wound healing complication ( $\mathrm{P}=0.19$ and 0.15 , respectively).

Of the 246 cases, the questionnaire survey was provided to the 109 patients with a follow-up longer than 1 year. Out of these patients, 54 responded to the questionnaire survey (Tables 4, 5). The mean follow-up for this subset group was 32.4 months.

\section{Table 2. Questionnaire}

1. Do you feel abnormal pain at the operation site of the toe?

2. Does the pain come with specific activities or situations?

2-1. What specific activities or situations are involved?

$\rightarrow$ Walking, running, ascending stairs, standing for a long time, working

3. Can you score your pain of the toe that underwent the operation? $0=$ no pain at all

$10=$ worst pain imaginable

4. How would you explain the pain?

5. Does your pain have any of features listed below? $\rightarrow$ Burning, painful cold, electric shocks

6. Is the pain associated with one or more of following symptoms in the same area? $\rightarrow$ Tingling, pins and needles, numbness, itching

7. How does the pain affect your daily activities? $\rightarrow$ No, somewhat, to a large extent

8. Have you taken a pain killer because of the pain?

9. Are you unable to walk or need a cane while walking?

10. Since the operation, have you had any abnormality of the toe while performing daily activities?

11. Since the operation, have you had any abnormality of the toe at your work-place?

12. How satisfied are you with the appearance of your toe now? $\rightarrow$ Good, acceptable, poor 


\section{Fig. 1. Harvest of toe pulp free flap}

Surgical technique of flap harvest from left second toe. (A) Design of the flap. (B) The subcutaneous vein entering the flap is identified at the base of the flap. (C) With flap dissection by separating vertical fibers, the digital artery is exposed along the medial side of the flap. (D) The flap including the neurovascular pedicle is isolated. (E) The harvested flap. (F) The donor site is closed primarily.
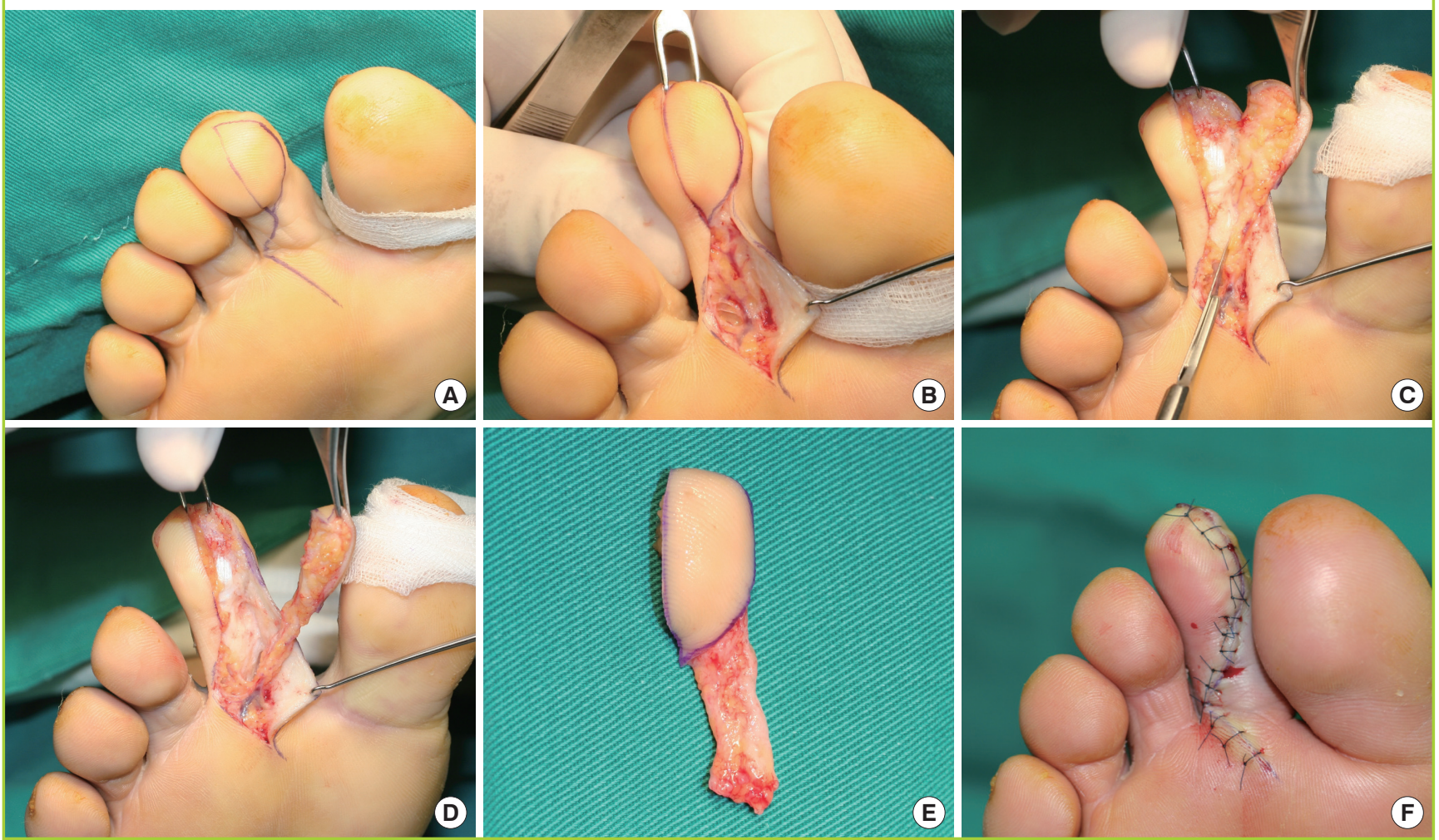

\section{Fig. 2. Coronal section of harvested flap}

We have usually performed flap elevation of no more than $50 \%$ of the arc of toe pulp as assessed coronally. The flap harvest sometimes necessitated taking up to $60 \%$ of the toe pulp arc, over the midline of the arc.

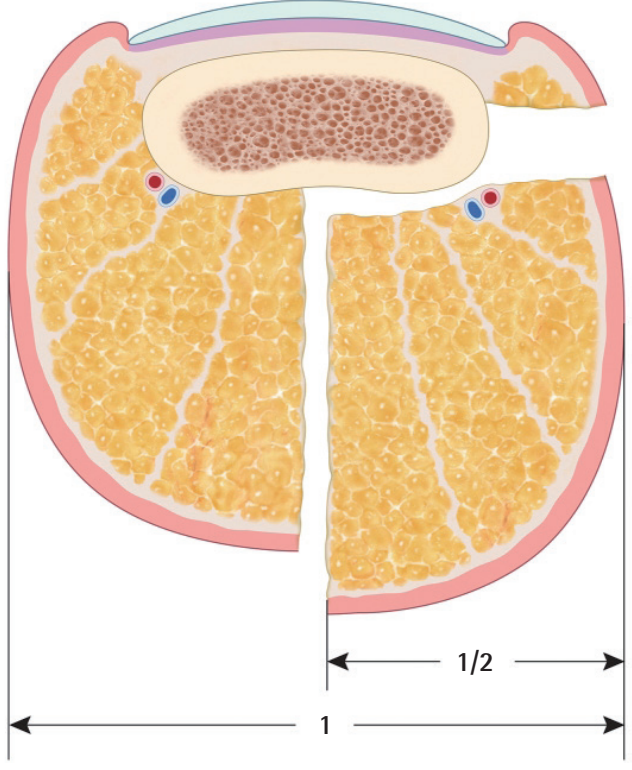

Harvested flap sizes ranged from $30 \%$ to $60 \%$ of the plantar surface of the second toe. Pain persisting beyond 1 year was present in 15 out of 54 donor sites (27.8\%). The Semmes-Weinstein monofilament test identified hypoesthesia of the donor site in 3 cases $(5.6 \%)$. Two of these patients had decreased protective sensation, with the remaining patients demonstrating a decrease in light touch sensation. Harvested flap size was not associated with donor-site sensory disturbance $(P=0.298)$. None of the patients had any nail deformity or hypertrophic scar in the donor toe. Neither pre-existing medical conditions nor smoking history was associated with sensory disturbance $(P=0.346$, $0.553)$. Most patients described postoperative donor-site appearance as 'good' $(n=38,70.4 \%)$, followed by 'acceptable' $(n=$ $13,24.1 \%)$ and 'poor' $(n=3,5.5 \%)$.

Among the 15 patients who described donor-site pain, pain intensity was distributed towards the lower end of the pain scale (pain scores from 2 to 5). The donor-site pain was described as stabbing $(n=8)$, electric shock (4), heaviness (2), and cold intolerance (1). None of the patients reported neuropathic pain above 2 points on the 7-item DN4 scale. Eleven of the patients reported activity associated with pain, such as walking $(n=7)$, standing still (3), and running (1). Six patients reported the do- 
Table 3. Patient data and early donor site complications

\begin{tabular}{|lc|}
\hline Criteria & Value \\
\hline No. of patients & 246 \\
Mean age (yr) & 41.2 \\
Sex (male:female ratio) & $204: 42$ \\
Mean flap width (cm) & 1.5 \\
Mean flap length (cm) & 2.5 \\
Smoking:non-smoking & $114: 132$ \\
Diabetes & 8 \\
Hypertension & 23 \\
Early donor site complications & \\
Infection & 0 \\
Skin necrosis & 0 \\
Wound dehiscence & 8 \\
Hematoma & 5 \\
\hline
\end{tabular}

Table 4. Patient data of long-term donor site assessment

\begin{tabular}{|lc|}
\hline Criteria & Value \\
\hline No. of patients & 54 \\
Mean age (yr) & 41.7 \\
Mean follow-up period (mo) & 32.4 \\
Sex (male:female ratio) & $43: 11$ \\
Smoking:non-smoking & $21: 33$ \\
Diabetes & 2 \\
Hypertension & 6 \\
Percentage of harvested flap from the entire toe pulp & \\
$\quad$ Minimum (\%) & 30 \\
Maximum (\%) & 60 \\
$\quad$ Average (\%) & 44.3 \\
Mean flap width (cm) & 1.4 \\
Mean flap length (cm) & 2.5 \\
\hline
\end{tabular}

nor-site pain to be limiting daily activity 'somewhat', while the remaining 9 patients described the pain as having 'no' effect on daily activity. None of the patients were taking prescription or over-the-counter pain medications.

\section{DISCUSSION}

Owing to the analogous nature of fingers and toes, the partial second toe pulp free flap offers the most natural replacement tissue for fingertip defects. The idea of using a portion of the second toe pulp to resurface fingertips can be traced back to several microsurgical pioneers in the 1970s. Over the ensuing 40 years, second toe pulp free flaps have found a niche indication for fingertip loss at select hand surgery and microsurgical replantation centers, with evolving standards of care.

Initially, interest in these partial pulp free flaps has focused on technical feasibility. Buncke and Rose [7] reported a series of six patients undergoing complete toe flap transfer in which the first two flaps were lost due to vascular congestion - with a flap survival rate of $67 \%$. With advancements in microsurgical techni-

\section{Table 5. Long-term assessment of donor site}

\begin{tabular}{|lc|}
\hline Assessment criteria & No. (\%) \\
\hline Pain & $15(27.8)$ \\
Average NRS score & 2.9 \\
Neuropathic feature & 0 \\
Degree of effect on daily activity & 9 \\
$\quad$ No effect & 6 \\
Somewhat & 0 \\
To a large extent & 0 \\
Pain medication & $3(5.6)$ \\
Sensory disturbance & 1 \\
Diminished light touch & 2 \\
Diminished protective sensation & 0 \\
Loss of protective sensation & 0 \\
Nail deformity & 0 \\
Gait disturbance & \\
Appearance & 38 (70.4) \\
Good & $13(24.1)$ \\
Acceptable & $3(5.5)$ \\
Poor & \\
\hline NRS, numerical rating scale (from 0, no pain; to 10, worst pain imaginable). \\
\hline
\end{tabular}

ques and flap physiology, this loss rate was shown to be an issue of whether the surgeon and the microsurgical center were able to provide the necessary technical expertise and postoperative care. In our previous publication regarding this subject, we reported 929 cases of partial second toe pulp free flaps [1]. Among these, 72 flaps required secondary interventions such as resection of a spastic arterial portion and/or vein grafting. Additionally, 58 flaps required leech therapy for venous congestion. Vigilant postoperative surveillance for flap failure and physiologically appropriate intervention made possible a flap survival rate of $99.7 \%$ (926/929). Previous to the introduction of second toe pulp free flaps, the academic debate had been how much sensation a given technique could restore to a fingertip with a pulp defect. The initial case series from Buncke and Rose revealed two-point discriminatory resolutions of 3 to $5 \mathrm{~mm}$-offering the promise of complete sensory restoration. Subsequent to this, Ratcliffe and McGrouther [12] reported a series of 13 toe pulp transfer cases in which the mean discriminatory resolution was $9.6 \mathrm{~mm}$. In our experience of over one thousand transfer flaps, the mean resolution was found to be $8 \mathrm{~mm}$, with younger patients ( $<35$ years) discriminating to $6 \mathrm{~mm}$.

With maturation of toe pulp flap techniques and postoperative management, the attention regarding surgical outcomes should turn from the recipient fingertip to the donor toe. In our previous publication, we had reported postoperative hematoma (39) and wound dehiscence (20) as postoperative complications. This early donor-site complication rate (59/929, 6\%) is comparable to that of the current study (13/246, 5.3\%).

In terms of long-term postoperative pain and daily activity, the questionnaire allowed for a detailed observation of the impact 
Fig. 3. Donor-site appearance

(A, B) Donor-site appearance 29 months after surgery.
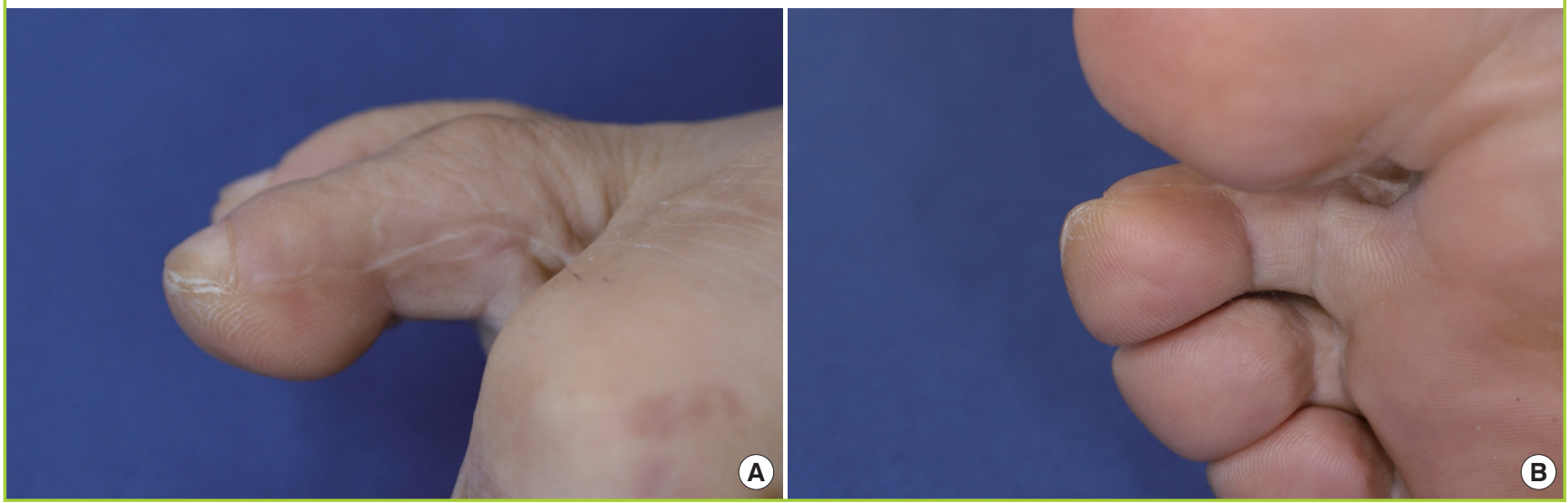

of partial pulp harvest. Initially, we were somewhat surprised at

donor-site morbidity during the informed consent process. the fact that more than $25 \%$ of the patients completing the survey continued to have pain more than a year after the operation $(15 / 54,27.8 \%)$. However, a close look at the remainder of the questionnaire survey revealed that the pain was described as being less than 5 on a $0-10$ intensity scale. Most of the patients who complained of long-term postoperative pain found that the pain was intermittent and did not interfere with daily activity. Additionally, none of the patients with long-term donor-site pain felt that pain medications were necessary. The donor-site appearance was satisfactory to most patients (Fig. 3).

This study has several limitations, owing to its retrospective design. Patients who voluntarily participated in the questionnaire may have over-represented the subset population with worse outcomes and may have contributed to the higher percentage of patients expressing pain. Additionally, the overall study population was not identical to the population of patients from our previous publication, though immediate postoperative wound complications ( $6 \%$ vs. $5.3 \%$ ) were comparable. The most significant limitation was that the study did not evaluate donor-site morbidities in relation to recipient-site gains. The survey could have included a subjective assessment on whether a patient felt that the tradeoff between the donor toe and recipient finger was a fair one. In future research, the etiology of wound dehiscence and hematoma should be explored, including any correlation to the size of the harvested flap.

The partial second toe pulp flap is associated with low rates of wound complications. Long-term postoperative pain was present in about a quarter of the patients surveyed. This pain neither required any pain medication nor interfered with daily activity. Given the functional and aesthetic gain in the recipient finger, donor-site morbidity appears acceptable in this patient population. This study can be helpful in counseling patients regarding

\section{REFERENCES}

1. Lee DC, Kim JS, Ki SH, et al. Partial second toe pulp free flap for fingertip reconstruction. Plast Reconstr Surg 2008; 121:899-907.

2. Bickel KD, Dosanjh A. Fingertip reconstruction. J Hand Surg Am 2008;33:1417-9.

3. Lister G. Local flaps to the hand. Hand Clin 1985;1:621-40.

4. Thoma A, Vartija LK. Making the V-Y advancement flap safer in fingertip amputations. Can J Plast Surg 2010;18:e47-9.

5. O’Brien B. Neurovascular island pedicle flaps for terminal amputations and digital scars. Br J Plast Surg 1968;21:258-61.

6. Cohen BE, Cronin ED. An innervated cross-finger flap for fingertip reconstruction. Plast Reconstr Surg 1983;72:688-97.

7. Buncke HJ, Rose EH. Free toe-to-fingertip neurovascular flaps. Plast Reconstr Surg 1979;63:607-12.

8. Logan A, Elliot D, Foucher G. Free toe pulp transfer to restore traumatic digital pulp loss. Br J Plast Surg 1985;38:497500.

9. Williamson A, Hoggart B. Pain: a review of three commonly used pain rating scales. J Clin Nurs 2005; 14:798-804.

10. Remerand F, Godfroid HB, Brilhault J, et al. Chronic pain 1 year after foot surgery: epidemiology and associated factors. Orthop Traumatol Surg Res 2014;100:767-73.

11. Bouhassira D, Attal N, Alchaar H, et al. Comparison of pain syndromes associated with nervous or somatic lesions and development of a new neuropathic pain diagnostic questionnaire (DN4). Pain 2005;114:29-36.

12. Ratcliffe RJ, McGrouther DA. Free toe pulp transfer in thumb reconstruction: experience in the West of Scotland Regional Plastic Surgery Unit. J Hand Surg Br 1991;16:165-8. 